\title{
Other and Unspecified Parts of Mouth ICD-O-3
}

National Cancer Institute

\section{Source}

National Cancer Institute. Other and Unspecified Parts of Mouth ICD-O-3. NCI

Thesaurus. Code C148343.

Components of the mouth that are referenced in topographic category C06 of ICD-O-3. 